\title{
Performance of a Novel Detector for TEM Employing a 4T Pixel Design for On- Chip Correlated Double Sampling and a Selectable Full-Well Capacity
}

\author{
Marco Oster ${ }^{1}$, Reza Ghadimi ${ }^{1}$, Hans Tietz ${ }^{1}$ \\ 1. Tietz Video and Image Processing Systems, Gauting, Germany
}

Former scintillator based CMOS camera systems with a classical three transistor (3T) pixel design already proved their usefulness several years ago [1] and may still have the edge over direct detection sensors for certain applications, such as low voltage microscopy [2] and imaging conditions with high beam current densities [3]. By leveraging recent progress of the semiconductor industry, manufacturing of an updated sensor with a $4 \mathrm{~T}$ pixel design is now feasible for the large sensor dimensions required in TEM. We report on the performance of such a novel scintillator based CMOS detector with a selectable full-well capacity for optimizing both, low noise and high dynamic range applications, on-chip correlated double sampling for reduced kTC (or reset) noise and a high frame rate at true 16 bit digitization.

As TEM may produce images with very different characteristics, e.g. huge contrast in diffraction mode or very faint intensity in low-dose imaging of delicate samples, a camera system with the ability to adapt to the imaging conditions is highly desirable. Each pixel of the novel sensor has an additional capacitor which allows the camera to be read out in either a high sensitivity or an extended dynamic range mode, where the effective full-well capacity is tripled. This allows optimizing the detector's response for faithful recording of the contrast conditions, as shown for a diffraction image in Figure 1.

The used silicon manufacturing process for the novel sensor enables implementing a floating diffusion node which collects the signal charge generated in a pinned photo diode. This enables on-chip correlated double sampling, where the reset potential is subtracted from the image signal with a very good time correlation and thus effectively eliminating the sampled kTC noise in both, rolling shutter and single exposure mode. The effect can be seen in Figure 2A, which clearly shows single electron events above the background noise level for a frame taken in rolling shutter mode.

Due to the high signal-to-noise ratio and small readout time in rolling shutter mode, single electron counting experiments become feasible with this scintillator based CMOS camera system (cf. Figures 2B, 2C). Since the electron sensitive surface is directly exposed to the electron beam, single electron events can be recorded even for accelerating voltages as low as $20 \mathrm{kV}$.

\section{References:}

[1] M Stumpf et al, Microsc. Microanal. 16 Suppl 2 (2010), p. 856.

[2] C Kealhofer et al, Ultramicroscopy 159 (2015), p. 19-25.

[3] JA Rodriguez and T Gonen in "Methods in Enzymology Vol. 579", ed. RA Crowther, (Academic Press, Burlington) p. 369-392. 

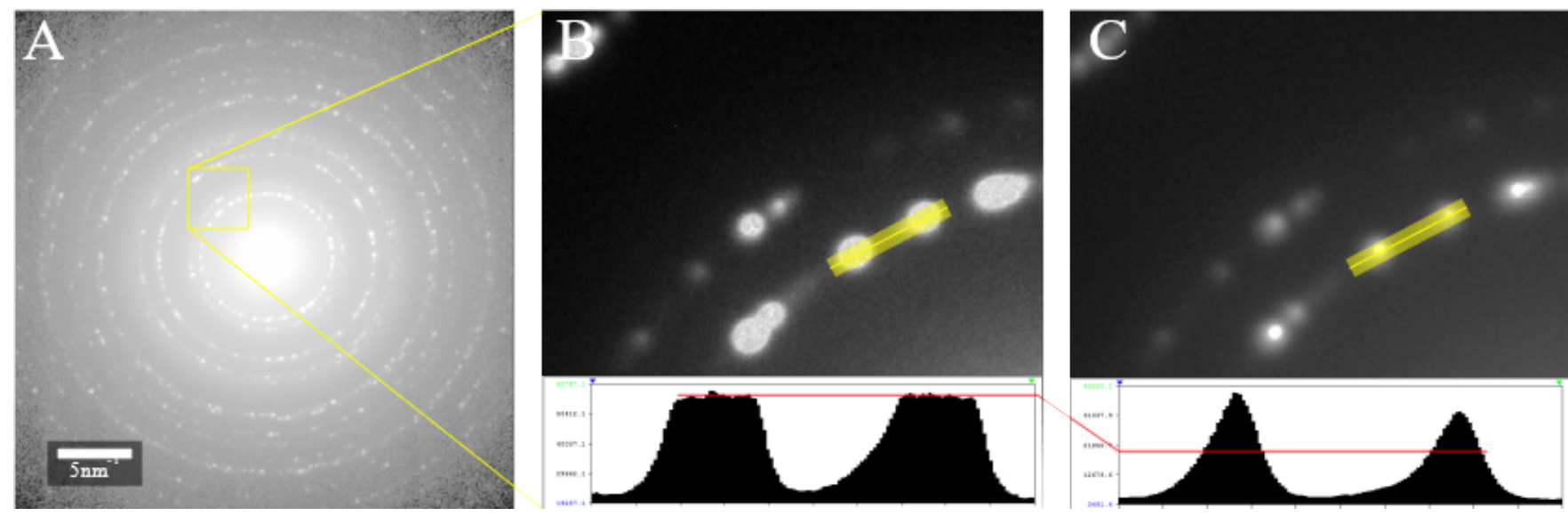

Figure 1. Switchable full-well capacity. Diffraction pattern of polycrystalline Al (A) with details imaged in high sensitivity (B) and extended dynamic mode (C). The line scans in the insets show pixel saturation in (B) but not in (C). Equivalent dose potentials are marked in the line scan diagrams. All images have been taken with identical illumination conditions and exposure times.
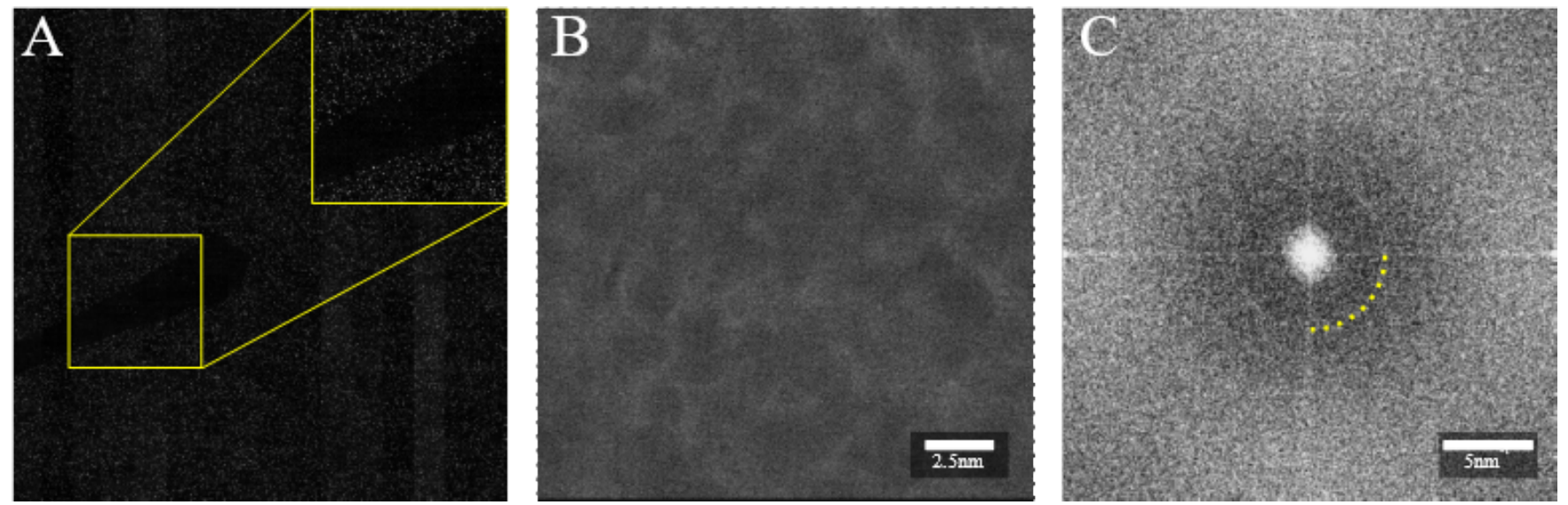

Figure 2. Single electron events at $200 \mathrm{kV}$. Single frame recorded in rolling shutter mode with a frame time of $40 \mathrm{~ms}$, illuminated with a very low current density and inserted beamstop (A), magnified in the inset. The single electron events are clearly visible and well contained to a $3 \times 3 \mathrm{px}$ area. Reconstructed single electron events are integrated to form an image of polycrystalline $\mathrm{Au}(\mathrm{B})$. With a total dose of only 0.6 electrons per pixel, the $\mathrm{Au} 111$ distance is already visible in the power spectrum (C). 